\title{
Repeat thermal ablation for local progression of lung tumours: how safe and efficacious is it?
}

\author{
Henry Zhao ${ }^{1,2}$, Satomi Okano ${ }^{3}$, Anita Pelecanos ${ }^{3}$, Karin Steinke , $^{1,2}$ \\ 'Department of Medical Imaging, Royal Brisbane and Women's Hospital, Brisbane, QLD 4029, Australia. \\ ${ }^{2}$ School of Medicine, The University of Queensland, Brisbane, QLD 4006, Australia. \\ ${ }^{3}$ QIMR Berghofer Medical Research Institute, Brisbane, QLD 4006, Australia.
}

Correspondence to: Prof. Karin Steinke, Department of Medical Imaging, Royal Brisbane and Women's Hospital, Bowen Bridge Rd \& Butterfield St, Herston, Brisbane, QLD 4029, Australia. E-mail: karin.steinke@gmail.com

How to cite this article: Zhao H, Okano S, Pelecanos A, Steinke K. Repeat thermal ablation for local progression of lung tumours: how safe and efficacious is it? Mini-invasive Surg 2018;2:26. http://dx.doi.org/10.20517/2574-1225.2018.27

Received: 24 May 2018 First Decision: 3 Jul 2018 Revised: 19 Jul 2018 Accepted: 2 Aug 2018 Published: 28 Aug 2018

Science Editor: Noriyoshi Sawabata Copy Editor: Yuan-Li Wang Production Editor: Huan-Liang Wu

\begin{abstract}
Aim: To retrospectively evaluate the safety and efficacy of repeat thermal ablation for local progression of lung tumours after prior ablation(s).

Methods: From December 2009 to March 2017, 13 patients underwent repeat ablation [11 repeat microwave ablations and 2 repeat radiofrequency ablations] of a lung tumour [9 non-small cell lung carcinomas, 3 metastatic colorectal adenocarcinomas, 1 metastatic pelvic sarcoma] for local progression after prior ablation(s). Safety of the procedure was assessed by presence or absence of adverse events. Efficacy of the procedure was assessed by local tumour response to ablation and survival time.
\end{abstract}

Results: Repeat ablation procedures were safe, without major adverse events. Median length of hospital stay was 2 days (interquartile range 1-2). Pneumothorax was the most common complication [5 (38\%) of 13 repeat ablation procedures]. There was one death within 30 days of ablation, but the cause of death and its relation to the procedure were unknown. Of the 12 patients with imaging follow-up [median follow-up 26 months (range 3-62)], 10 (83\%) had complete ablation and $2(17 \%)$ had local progression. Of all 13 patients, $8(62 \%)$ were alive and $5(38 \%)$ had died with a median overall survival of 43 months (95\% confidence interval 36-49 months).

Conclusion: Repeat ablation in locally progressing tumours after prior ablation attempt(s) is a safe therapeutic option and often achieves local tumour control.

Keywords: Local progression, lung tumours, microwave ablation, radiofrequency ablation, repeat ablation

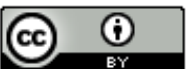

(C) The Author(s) 2018. Open Access This article is licensed under a Creative Commons Attribution 4.0 International License (https://creativecommons.org/licenses/by/4.0/), which permits unrestricted use, sharing, adaptation, distribution and reproduction in any medium or format, for any purpose, even commercially, as long as you give appropriate credit to the original author(s) and the source, provide a link to the Creative Commons license, and indicate if changes were made. 


\section{INTRODUCTION}

CT-guided thermal ablation has developed in the last 20 years as a minimally invasive management option for pulmonary malignancies, both primary and secondary, in patients who are non-surgical candidates ${ }^{[1]}$. Radiofrequency ablation (RFA), an electric current-based heating technique, has been the most evaluated $\operatorname{method}^{[2]}$. Microwave ablation (MWA), which uses an electromagnetic field around the ablation device to create heat, is a more updated technique with theoretical advantages in terms of lesser susceptibility to heat-sink effect, larger ablations, more uniform ablation zones, and shorter procedural times ${ }^{[2,3]}$. Ablation failure and subsequent local tumour progression remain an issue for both methods, with tumour diameter greater than $3 \mathrm{~cm}$, proximity to a large vessel and insufficient ablative margin being risk factors for failure $^{[2,4]}$. There are limited data on the role of repeat ablation for local progression of lung tumours after prior ablation, and with most data on repeat $\mathrm{RFA}^{[5-7]}$. This retrospective cross-sectional study aimed to evaluate the safety and efficacy of repeat MWA and RFA, in locally progressing lung tumours after prior ablation(s) at a large tertiary hospital.

\section{METHODS}

\section{Patients}

Ethics approval for this study was obtained from the Hospital Institutional Review Board (HREC/14/ QRBW/553). Patients were identified using the radiology thermal ablation database at our large tertiary hospital. Data were collected retrospectively through systematic review of patient charts and imaging. Inclusion criteria were consecutive patients who underwent repeat percutaneous CT-guided thermal ablation of a lung tumour for local progression after prior ablation(s) between December 2009 and March 2017. There were no exclusion criteria for this study. Repeat ablation was defined as the most recent ablation procedure, after prior ablation(s). All lesions were referred for ablation if patients are medically inoperable or refuse surgery. Patients were informed of the risks and benefits of ablation, and all provided written informed consent for the procedure.

\section{Ablation procedure and imaging follow-up}

Ablations were performed percutaneously under CT guidance (Philips Brilliance 64, Eindhoven, The Netherlands, replaced in 2015 by Aquilion ONE, Toshiba Medical Systems, Otawara, Tochigi, Japan). Both RFA and MWA modalities were used (with MWA having replaced RFA at our institution by mid-2010). All ablations were performed by the same interventional radiologist who had more than 15 years' experience in image-guided ablation. No prophylactic antibiotic treatment was administered. The ablation applicator was inserted under sterile conditions under CT guidance, with analgesia and sedation administered by the anaesthetic team. Parameters such as the type of applicator, number of overlapping ablations, ablation duration, and amount of energy delivered were based on individual tumour size, location and tumour characteristics. Patients received a chest X-ray $3 \mathrm{~h}$ post-procedure to assess for post-procedural complications, and in all MWA cases, a limited non-contrast CT scan of the ablated site was performed $24 \mathrm{~h}$ post-procedure. Technically successful ablation was defined as completed planned ablation cycle(s) and circumferential perilesional ground-glass opacity. Patients were requested to have serial repeat CT scanning at 3, 6 and 12 months post-ablation and 6 monthly scans thereafter to evaluate treatment outcome. An FDG-PET scan was also requested at 6 months post-ablation.

\section{Outcomes}

Safety of the procedure was assessed by presence or absence of adverse events. Adverse events were recorded and classified in accordance with the Common Terminology Criteria for Adverse Events version $4.03^{[8]}$.

Efficacy of the procedure was assessed by local tumour response to ablation and survival time. For MWA, the limited CT scan of the ablated site $24 \mathrm{~h}$ post-ablation served as the baseline scan that all subsequent follow-up scans were compared against; for RFA, the immediate post-ablation scan was used. Local progres- 


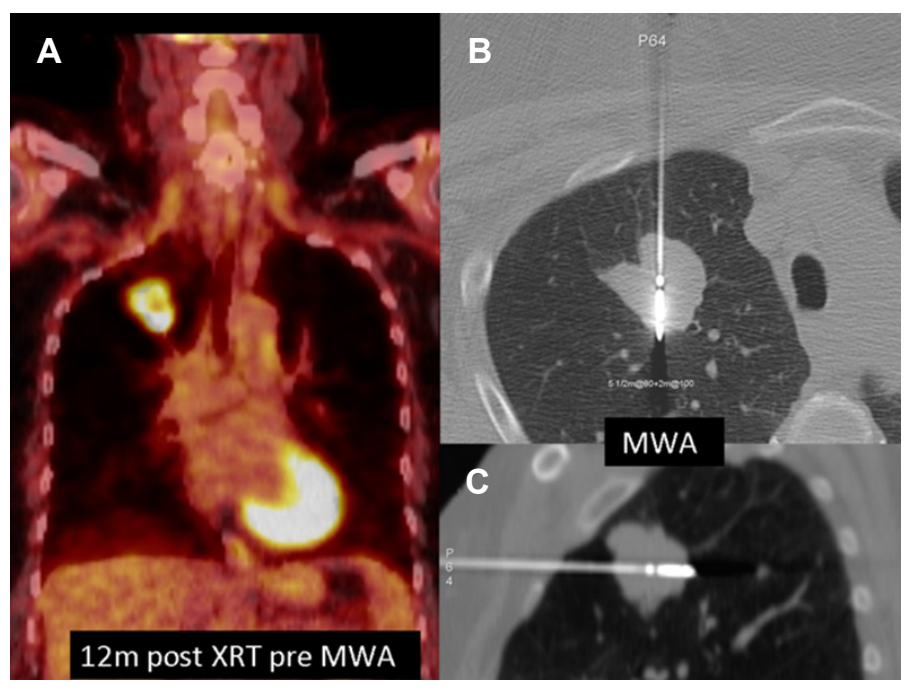

Figure 1. 57-year-old female with RUL SCC. A: Coronal FDG-PET scan 12 months post definitive radiotherapy (58 Gy) RUL T2NO SCC; B, C: microwave antenna (Acculis standard pMTA) centrally within the tumour; a total of 7 min $30 \mathrm{~s}$ ablation time-5 min $30 \mathrm{~s}$ at $80 \mathrm{~W}$ followed by 2 min at $100 \mathrm{~W}$. RUL: right upper lobe; SCC: squamous cell carcinoma

sion after initial ablation was defined as an increase in the size (maximum axial diameter) of the ablation zone on CT. Stability or decrease in size of the ablation zone on CT was considered complete ablation ${ }^{[9]}$. Patients were followed up until 30 Jun 2017. Patients were either alive, evidenced by a documented visit to an outpatient clinic, general practitioner or scan appointment after this date, or deceased, as recorded on the state health database. Follow-up periods were calculated from date of repeat ablation.

\section{Statistical methods}

The characteristics of patients and treatment outcomes were summarised using frequencies and percent for categorical variables and mean [standard deviation (SD)] or median [interquartile range (IQR) and range] for continuous variables. Kaplan-Meier survival estimates were produced for overall survival. STATA $15^{[10]}$ was used for analyses.

\section{RESULTS}

\section{Patient and tumour characteristics}

From December 2009 to March 2017, 13 patients (6 male, 7 female) underwent repeat ablation of a lung tumour due to local progression after prior ablation(s) [Figures 1-5]. All prior ablations had been technically successful. Mean age at repeat ablation was 72 years (SD 11, range 53-88) [Table 1].

The ablated lung tumours consisted of: non-small cell lung carcinoma (NSCLC) (8 stage 1 NSCLCs, and 1 multifocal bronchioloalveolar carcinoma with bilateral lung involvement), metastatic colorectal adenocarcinoma (3) and metastatic pelvic sarcoma (1). Four of the NSCLC lesions had locally progressed postradiotherapy and were subsequently referred for ablation.

\section{Thermal ablation characteristics}

Eleven of the 13 patients had repeat MWA; 7 patients after 1 prior MWA procedure, 2 patients after 2 prior MWA procedures, and 2 patients after 1 prior RFA treatment. The remaining 2 patients had repeat RFA after 1 prior RFA treatment. Nine of the 11 repeat MWAs were performed with the Acculis Microwave Tissue Ablation system (Angiodynamics, Latham, New York, USA), which operates at $2.45 \mathrm{GHz}$ with a maximum power output of 140 watts; a standard antenna with a $16 \mathrm{~mm}$ active tip was used in these 9 cases. The remaining 2 repeat MWAs were performed with the HS Amica microwave system (HS Amica, HS Hospital 


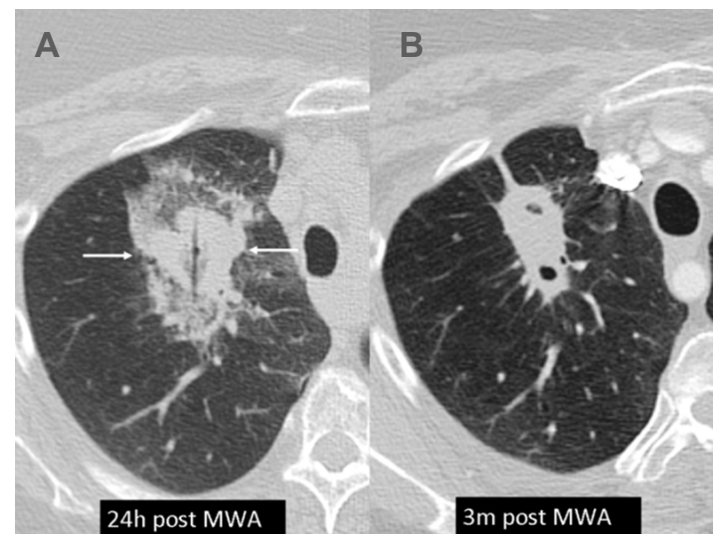

Figure 2. 57-year-old female with right upper lobe squamous cell carcinoma. Axial CT scan lung windows. A: 24 h post microwave ablation showing surrounding GGO, most narrow GGO zone medially and laterally (arrows); B: 3 months post microwave ablation showing satisfactory shrinking of ablation zone, small central cavities. GGO: ground-glass opacity

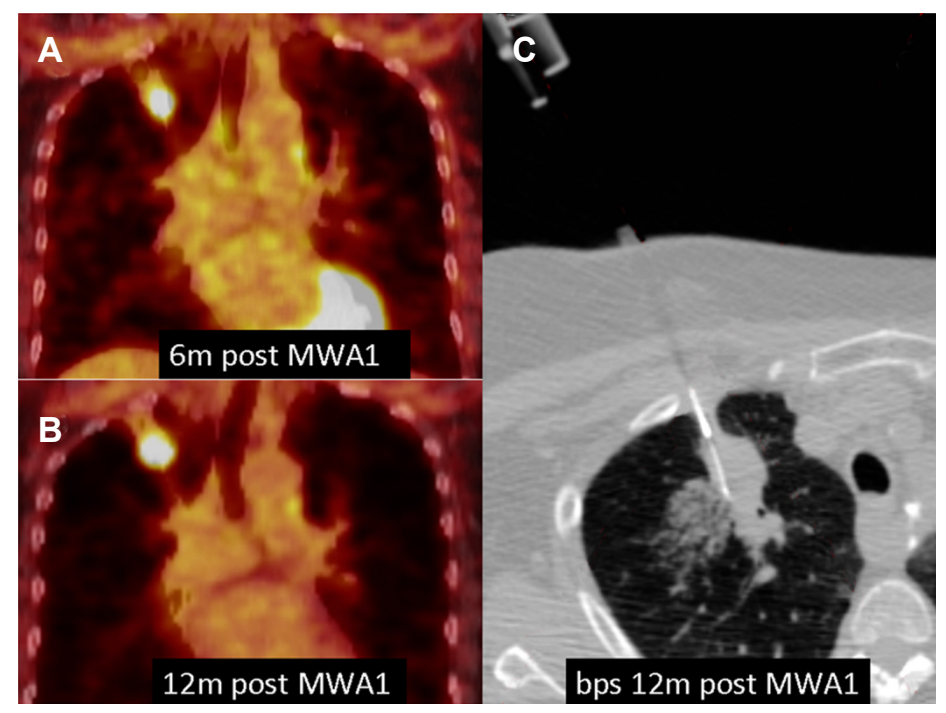

Figure 3. 57-year-old female with right upper lobe SCC. A, B: Coronal FDG-PET/CT scans 6 months and 12 months post microwave ablation showing residual FDG avidity increasing; C: CT guided core biopsy demonstrated local recurrence of SCC. CT guided core biopsy demonstrated local recurrence of SCC. SCC: squamous cell carcinoma

Service, Rome, Italy), which operates at $2.45 \mathrm{GHz}$ with a maximum power output of 140 watts; a 16 gauge $15 \mathrm{~cm}$ probe was used in both cases. Of the 2 patients treated with repeat RFA, the Cool-tip internally cooled electrode system (Cool-tip, Valleylab, Boulder, Colorado, USA) with a $3 \mathrm{~cm}$ active tip was used for one patient, and the Rita Starbust XL Electrosurgical device (Rita Medical Systems, Mountain View, California, USA, now Angiodynamics) was used for the other patient.

\section{Procedure tolerability and complications}

Repeat ablation procedures were generally safe, without major adverse events [Table 2]. Median length of hospital stay was 2 days (IQR 1-2, range 1-7) [Table 2]. All but 1 (8\%) of the repeat ablation procedures were technically successful. One procedure was complicated by intraprocedural pneumothorax, which enlarged during the procedure, prompting a request from the anaesthetic team to abort the procedure given the patient's comorbidities of chronic obstructive pulmonary disease on home oxygen, obstructive sleep apnoea and morbid obesity. The pneumothorax was aspirated twice during the procedure, and required chest tube 


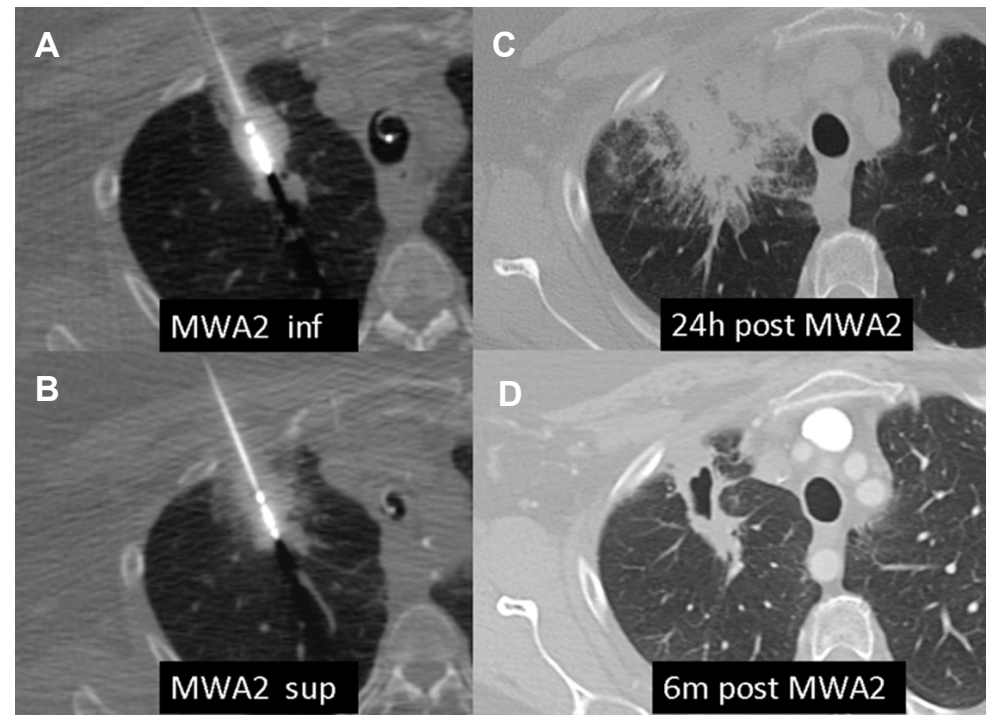

Figure 4. 57-year-old female with right upper lobe squamous cell carcinoma. A, B: Repeat microwave ablation, 2 overlapping ablation cycles; C: axial CT $24 \mathrm{~h}$ post re-ablation shows circumferential ground-glass opacity of at least $1 \mathrm{~cm}$; D: axial CT 6 months post reablation shows significant shrinking of ablation site with central cavitation

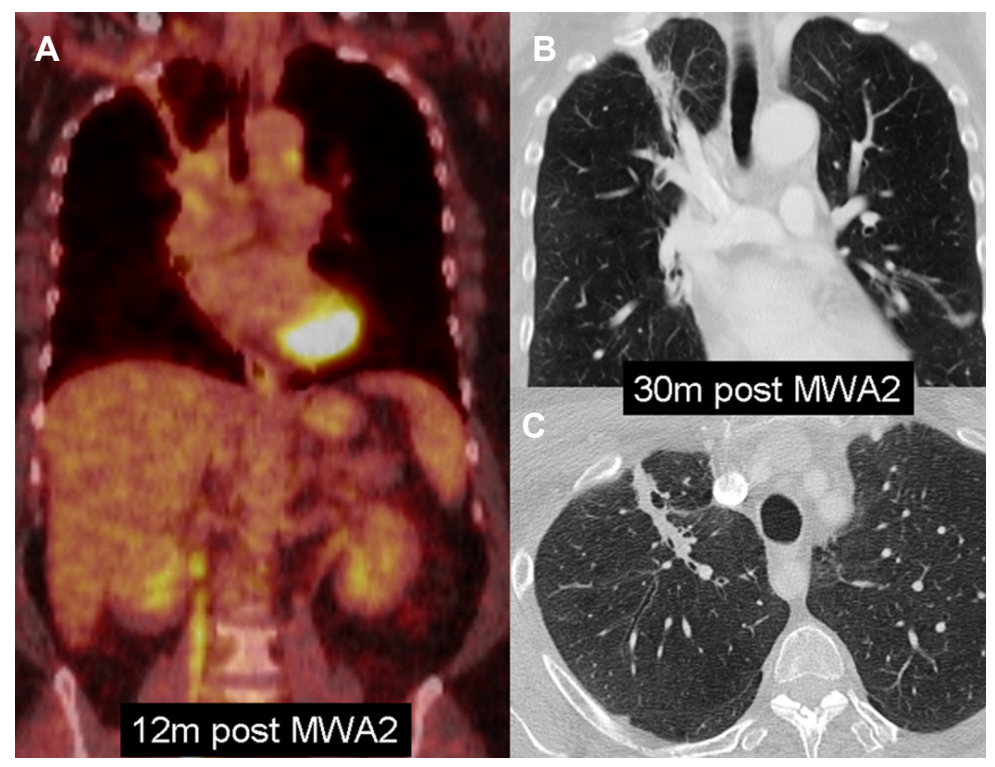

Figure 5. 57-year-old female with RUL squamous cell carcinoma. A: Coronal FDG-PET/CT shows no residual FDG uptake at the RUL ablation site 12 months post re-ablation; B, C: coronal and axial CT scan lung windows 30 months post re-ablation shows band-like scarring/atelectasis at site of previous tumour. RUL: right upper lobe

insertion after the procedure, which resolved the pneumothorax. The patient subsequently had an uneventful recovery.

There were no intraprocedural deaths. Five (38\%) patients experienced pneumothorax from the procedure, $3(23 \%)$ of whom required chest tube insertion [Table 2]. There was $1(8 \%)$ death within 30 days of the procedure (repeat ablation of metastatic pelvic sarcoma), although the cause of death and relation to the procedure were unknown. The patient had been discharged the day after an uneventful ablation procedure and died at home two days after the procedure of a sudden death during the night ${ }^{[11]}$. The presumed cause 
Table 1. Patient and lung tumour characteristics at repeat ablation

\begin{tabular}{ll}
\hline & Total $(\boldsymbol{n}=\mathbf{1 3})$ \\
\hline Age (years), Mean (SD) & $72(11.0)$ \\
Sex & \\
Female & $7(54)$ \\
Male & $6(46)$ \\
Tumour type & \\
Non-small cell lung carcinoma & $9(69)$ \\
Colorectal adenocarcinoma & $3(23)$ \\
Pelvic sarcoma & $1(8)$ \\
Type of prior ablation/s & \\
MWA & $9(69)$ \\
RFA & $4(31)$ \\
Number of prior ablation/s & \\
1 & $11(85)$ \\
2 & $2(15)$ \\
Radiotherapy prior to ablations & \\
No & $9(69)$ \\
Yes & $4(31)$ \\
Lobe & \\
LLL & $2(15)$ \\
LUL & $8(62)$ \\
RLL & $1(8)$ \\
RUL & $2(15)$ \\
Repeat ablation modality & \\
MWA & $11(85)$ \\
RFA & $2(15)$ \\
Diameter (mm)', median (IQR) & $28.2(22.0-40.0)$ \\
Number of ablation cycles & \\
2 & $1(8)$ \\
3 & $8(62)$ \\
Maximum power used (Watts) & $4(31)$ \\
Total ablation duration (minutes) $(M W A, n=11)$, median (IQR) & \\
\hline & $3(27)$ \\
\hline & $8(73)$ \\
& $4.5(4.0-6.0)$ \\
\hline
\end{tabular}

Tongest axial diameter in millimetres, immediately prior to repeat ablation. Data are presented as $n$ (\%) for categorical variables and as mean [standard deviation (SD)] or median [interquartile range (IQR)] for continuous variables. MWA: microwave ablation; RFA: radiofrequency ablation; LUL: left upper lobe; LLL: left lower lobe; RUL: right upper lobe; RLL: right lower lobe

of death was pulmonary embolism or myocardial infarction, however autopsy was declined and the cause of death was not definitively determined ${ }^{[11]}$.

\section{Response to treatment}

Only 12 patients had follow-up imaging performed. The 1 patient without follow-up imaging had died 2 days after the procedure. Median length of CT follow-up was 26 months (range 3-62), and median length of FDG-PET follow-up was 6 months (range 0-26). Ten of twelve (83\%) patients had complete ablation from their repeat ablation procedure. Two of twelve (17\%) had local progression [Table 2], one of whom had the technically unsuccessful repeat ablation procedure. Time to first detection of local progression on imaging for the 2 patients with local progression were 8 and 27 months respectively.

Six of twelve (50\%) patients had nodal or distant metastasis on follow-up imaging. Four of these patients had subsequent chemotherapy (the 2 patients in the local progression group, and 2 patients in the complete ablation group). Median time to first detection of metastasis on imaging was 20 months (range 11-36).

\section{Survival}

Of all 13 patients, 8 (62\%) were alive [median follow-up 30 months (range 3-91)] and 5 (38\%) had died (me- 
Table 2. Repeat ablation treatment outcomes and complications

\begin{tabular}{ll}
\hline & Total $(\boldsymbol{n}=\mathbf{1 3})$ \\
\hline Technically successful & $1(8)$ \\
No & $12(92)$ \\
Yes & \\
Complications & $7(54)$ \\
No & $6(46)$ \\
Yes & 1 \\
Pneumothorax (CTCAE grade 1) & 1 \\
Pneumothorax (CTCAE grade 1) and death within 30 days of procedure (CTCAE grade 5) & 2 \\
Pneumothorax (CTCAE grade 2) & 1 \\
Pneumothorax (CTCAE grade 2) and subcutaneous emphysema & 1 \\
Pleural effusion (CTCAE grade 1) & $2(1-2)$ \\
Length of hospital stay (days) & $54.0(41.0-60.0)$ \\
Post-ablation diameter (mm) ${ }^{\dagger}$ & $38.5(25.3-49.9)$ \\
Diameter on latest follow-up (mm) $(n=12)$ & \\
Local tumour response to ablation $(n=12)$ & $10(83)$ \\
Complete ablation & $2(17)$ \\
Local tumour progression & \\
Nodal or distant metastasis on follow-up imaging $(n=12)$ & $6(50)$ \\
No & $6(50)$ \\
Yes & \\
Mortality & $8(62)$ \\
Alive & $5(38)$ \\
Deceased & \\
\hline
\end{tabular}

'For MWA, maximum axial diameter on $24 \mathrm{~h}$ post-ablation CT scan. For RFA, maximum axial diameter on immediate post-ablation CT scan. Data are presented as n (\%) for categorical variables and as median [interquartile range (IQR)] for continuous variables. MWA: microwave ablation; RFA: radiofrequency ablation; CTCAE: Common Terminology Criteria for Adverse Events.

dian time to death 37 months (range 2 days-43 months). Median overall survival was 43 months (95\% confidence interval 36-49).

In the 9 patients with NSCLC, 5 (56\%) were alive [median follow-up 30 months (range 30-91)] and 4 (44\%) had died [median time to death 38 months (range 27-43)]; all 4 had metastatic NSCLC at time of death. All were alive at 2 years.

All 3 patients with metastatic colorectal cancer were alive at the last date of follow-up [median follow-up 23 months (range 3-38)].

Local response to ablation and corresponding survival status at follow-up for all patients is summarised in Table 3.

\section{DISCUSSION}

We described the safety and efficacy of repeat ablation in a heterogeneous population of locally progressing lung tumours after prior ablation, including both primary and metastatic lesions. Repeat ablations were safe and well-tolerated, and often achieved local control despite local progression after prior technically successful ablation. Pneumothorax was the most common procedural complication, asymptomatic or manageable with chest tube insertion. Rate of pneumothorax requiring chest tube insertion (23\% of procedures) was similar to that of other studies $(11 \%-29 \%)^{[7,12-18]}$. Other reported complications of lung ablation include pain, post-ablation syndrome, pleural effusion, subcutaneous emphysema, pneumonia, bronchopleural fistula, pulmonary haemorrhage, haemoptysis, nerve injury and, rarely, death ${ }^{[7,11-18]}$. Our study had one 30-day post-procedural death, but the cause of death and relation to the procedure were unknown. Other studies have estimated the 30 -day mortality rate after thermal ablation to be $0 \%-3 \%^{[12-15,19]}$. 
Table 3. Local tumour response to ablation and survival status at follow-up

\begin{tabular}{|c|c|c|c|}
\hline \multirow{2}{*}{ Local response } & \multicolumn{2}{|c|}{ Survival status } & \multirow{2}{*}{ Total } \\
\hline & Alive & Dead & \\
\hline Complete ablation & 7 & 3 & 10 \\
\hline Local progression & 1 & 1 & 2 \\
\hline No imaging follow-up & 0 & 1 & 1 \\
\hline Total & 8 & 5 & 13 \\
\hline
\end{tabular}

While surgery is the treatment of choice in early stage NSCLC, many patients are not candidates for surgery due to comorbidities ${ }^{[1]}$. Percutaneous thermal ablation is a minimally invasive alternative treatment option with minimal impact on the uninvolved lung parenchyma and overall pulmonary function ${ }^{[20]}$. Our results demonstrate that even after failed initial attempt(s) at ablation, repeat ablation can achieve local control. Only 2 of our patients experienced local progression on follow-up imaging after repeat ablation. Two-year overall survival in our NSCLC patients after repeat ablation was $100 \%$. Yang et al. ${ }^{[7]}$ recently reported repeat MWA to be a safe and effective treatment for local progression of medically inoperable stage 1 NSCLC after initial MWA, with 21 of 24 (87.5\%) patients achieving local control after repeat MWA, and with median overall survival 41.5 months after first MWA, similar to those treated with single MWA and with no local progression (median overall survival 48 months) at their institution. Our results support these findings that repeat thermal ablation is a safe and effective treatment for local progression of stage 1 NSCLC after initial ablation.

Thermal ablation may also be used for local control of secondary lung metastases. The goal in such patients is to improve patient quality of life and prolong survival. In a series of 566 patients with 1037 lung metastases of various primary origin, RFA treatment resulted in a median overall survival of 62 months, and a 4 -year local control rate of $44.1 \%$, with patients re-treated with RFA safely up to four times ${ }^{[9]}$. Our small sample of metastatic colorectal cancer patients re-treated with MWA all achieved local control and were alive after a median follow-up time of 23 months. The minimally invasive nature and repeatability of ablation are of utility in local control of lung metastases.

Six out of 12 patients had nodal or distant metastasis on follow-up imaging, with 2 of these patients demonstrating local progression at the ablation site, suggesting systemic disease progression despite local control in the other four patients. We believe offering repeat ablation for local control is still of value, as it is a minimally invasive technique which may improve patient quality of life and prolong survival, without significant increase in morbidity.

In this study, we defined local tumour response to ablation as based on increase, stability or decrease in size of ablation zone on $\mathrm{CT}^{[9]}$. FDG-PET scan findings are more difficult to interpret, as local uptake may be due to post-treatment inflammatory changes rather than local tumour progression, and this effect may last up to 12 months after ablation ${ }^{[21-23]}$. Additionally, false positive FDG-PET scans have been reported greater than 12 months after ablation ${ }^{[23]}$. The optimal timing of FDG-PET scan after ablation is controversial and not yet defined ${ }^{[24]}$. FDG-PET is likely a useful adjunct to CT in assessing local response to ablation, as well as for diagnosis of nodal or distant metastasis. However, further research is needed in imaging criteria to determine treatment response to ablation.

This study was limited by small sample size, heterogeneity of tumour size and histology, difference of ablation systems used and retrospective assessment. Additionally, as some patients had to travel long distances for their procedure and were not followed up by the respective teams they had been admitted under for their ablations, follow-up CT and FDG-PET scans were occasionally not performed at the requested time, although local tumour response to ablation was still able to be determined based on the most recent follow-up CT. Furthermore, some patients who had nodal or distant metastasis on follow-up imaging subsequently had chemotherapy, which may have influenced local response to ablation. In the future, larger 
studies should evaluate the effect of tumour type, and the use of other treatments such as chemotherapy, on response to repeat ablation.

In conclusion, despite these limitations, our study demonstrates that repeat MWA or RFA in locally progressing tumours after initial ablation attempt(s) may be generally safe, well tolerated, and often achieves local control. These findings should be validated in larger prospective studies.

\section{DECLARATIONS}

\section{Acknowledgments}

The authors would like to thank the Department of Nuclear Medicine at the Royal Brisbane and Women's Hospital for their assistance with FDG-PET imaging.

\section{Authors' contributions}

Design, literature research, data acquisition, drafted manuscript: Zhao H, Steinke K

Data analysis: Zhao H, Okano S, Pelecanos A, Steinke K

Substantively revised manuscript: Zhao H, Okano S, Pelecanos A, Steinke K

\section{Availability of data and materials}

Patients were identified using the radiology thermal ablation database at the Royal Brisbane and Women's Hospital. Data were collected retrospectively through systematic review of patient charts and imaging. The de-identified data is available by contacting the corresponding author Associate Professor Karin Steinke.

\section{Financial support and sponsorship}

None.

\section{Conflicts of interest}

All authors declared that there are no conflicts of interest.

\section{Ethical approval and consent to participate}

Ethics approval for this study was obtained from the Royal Brisbane and Women's Hospital Human Research Ethics Committee (HREC/14/QRBW/553).

\section{Consent for publication}

All patients provided written, informed consent.

\section{Copyright}

(c) The Author(s) 2018.

\section{REFERENCES}

1. de Baere T, Tselikas L, Gravel G, Deschamps F. Lung ablation: best practice/results/response assessment/role alongside other ablative therapies. Clin Radiol 2017;72:657-64.

2. Palussière J, Catena V, Buy X. Percutaneous thermal ablation of lung tumors - radiofrequency, microwave and cryotherapy: where are we going? Diagn Interv Imaging 2017;98:619-25.

3. Sidoff L, Dupuy DE. Clinical experiences with microwave thermal ablation of lung malignancies. Int J Hyperthermia 2017;33:25-33.

4. Ahmed M, Solbiati L, Brace CL, Breen DJ, Callstrom MR, Charboneau JW, Chen MH, Choi BI, de Baère T, Dodd GD 3rd, Dupuy DE, Gervais DA, Gianfelice D, Gillams AR, Lee FT Jr, Leen E, Lencioni R, Littrup PJ, Livraghi T, Lu DS, McGahan JP, Meloni MF, Nikolic B, Pereira PL, Liang P, Rhim H, Rose SC, Salem R, Sofocleous CT, Solomon SB, Soulen MC, Tanaka M, Vogl TJ, Wood BJ, Goldberg SN; International Working Group on Image-guided Tumor Ablation; Interventional Oncology Sans Frontières Expert Panel; Technology Assessment Committee of the Society of Interventional Radiology; Standard of Practice Committee of the Cardiovascular and Interventional Radiological Society of Europe. Image-guided tumor ablation: standardization of terminology and reporting criteria-a 10-year update. Radiology 2014;273:241-60.

5. Hiraki T, Mimura H, Gobara H, Sano Y, Fujiwara H, Date H, Kanazawa S. Repeat radiofrequency ablation for local progression of lung 
tumors: does it have a role in local tumor control? J Vasc Interv Radiol 2008;19:706-11.

6. Lanuti M, Sharma A, Willers H, Digumarthy SR, Mathisen DJ, Shepard JA. Radiofrequency ablation for stage I non-small cell lung cancer: management of locoregional recurrence. Ann Thorac Surg 2012;93:921-7.

7. Yang X, Ye X, Huang G, Han X, Wang J, Li W, Wei Z, Meng M. Repeated percutaneous microwave ablation for local recurrence of inoperable stage I nonsmall cell lung cancer. J Cancer Res Ther 2017;13:683-8.

8. National Cancer Institute. Common Terminology Criteria for Adverse Events, version 4.03. Available from: https://www.eortc.be/services/doc/ctc/CTCAE_4.03_2010-06-14_QuickReference_5x7.pdf. [Last accessed on 17 Aug 2018]

9. de Baère T, Aupérin A, Deschamps F, Chevallier P, Gaubert Y, Boige V, Fonck M, Escudier B, Palussiére J. Radiofrequency ablation is a valid treatment option for lung metastases: experience in 566 patients with 1037 metastases. Ann Oncol 2015;26:987-91.

10. StataCorp. Stata Statistical Software: Release 15. College Station, TX: StataCorp LLC 2017.

11. Splatt AM, Steinke K. Major complications of high-energy microwave ablation for percutaneous CT-guided treatment of lung malignancies: single-centre experience after 4 years. J Med Imaging Radiat Oncol 2015;59:609-16.

12. Schoellnast H, Deodhar A, Hsu M, Moskowitz C, Nehmeh SA, Thornton RH, Sofocleous CT, Alago W Jr, Downey RJ, Azzoli CG, Rosenzweig KE, Solomon SB. Recurrent non-small cell lung cancer: evaluation of CT-guided radiofrequency ablation as salvage therapy. Acta Radiol 2012;53:893-9.

13. Von Meyenfeldt EM, Prevoo W, Peyrot D, Lai A Fat N, Burgers SJ, Wouters MW, Klomp HM. Local progression after radiofrequency ablation for pulmonary metastases. Cancer 2011;117:3781-7.

14. Gillams A, Khan Z, Osborn P, Lees W. Survival after radiofrequency ablation in 122 patients with inoperable colorectal lung metastases. Cardiovasc Intervent Radiol 2013;36:724-30.

15. Yang X, Ye X, Zheng A, Huang G, Ni X, Wang J, Han X, Li W, Wei Z. Percutaneous microwave ablation of stage I medically inoperable non-small cell lung cancer: Clinical evaluation of 47 cases. J Surg Oncol 2014;110:758-63.

16. Steinke K, Glenn D, King J, Clark W, Zhao J, Clingan P, Morris DL. Percutaneous imaging-guided radiofrequency ablation in patients with colorectal pulmonary metastases: 1-year follow-up. Ann Surg Oncol 2004;11:207-12.

17. Acksteiner C, Steinke K. Percutaneous microwave ablation for early-stage non-small cell lung cancer (NSCLC) in the elderly: a promising outlook. J Med Imaging Radiat Oncol 2015;59:82-90.

18. Liu H, Steinke K. High-powered percutaneous microwave ablation of stage I medically inoperable non-small cell lung cancer: a preliminary study. J Med Imaging Radiat Oncol 2013;57:466-74.

19. Huang L, Han Y, Zhao J, Wang X, Cheng Q, Li X, Xu H, Gao K. Is radiofrequency thermal ablation a safe and effective procedure in the treatment of pulmonary malignancies? Eur J Cardiothorac Surg 2011;39:348-51.

20. Sofocleous CT, Sideras P, Petre EN, Solomon SB. Ablation for the management of pulmonary malignancies. AJR Am J Roentgenol 2011;197:W581-9.

21. McLoney ED, Isaacson AJ, Keating P. The role of PET imaging before, during, and after percutaneous hepatic and pulmonary tumor ablation. Semin Intervent Radiol 2014;31:187-92.

22. Yoo DC, Dupuy DE, Hillman SL, Fernando HC, Rilling WS, Shepard JA, Siegel BA. Radiofrequency ablation of medically inoperable stage IA non-small cell lung cancer: are early posttreatment pet findings predictive of treatment outcome? AJR Am J Roentgenol 2011;197:334-40.

23. Zaheer SN, Whitley JM, Thomas PA, Steinke K. Would you bet on PET? Evaluation of the significance of positive PET scan results post-microwave ablation for non-small cell lung cancer. J Med Imaging Radiat Oncol 2015;59:702-12.

24. Higuchi M, Suzuki H, Gotoh M. Role of PET/computed tomography in radiofrequency ablation for malignant pulmonary tumors. PET Clin 2016;11:47-55. 